\title{
Dual contraceptive method use in HIV-serodiscordant Kenyan couples
}

\author{
Alison C Roxby, ${ }^{1}$ Leïla Ben-Youssef, ${ }^{2,3}$ Grace Marx, $^{4}$ Freda Kinoti, ${ }^{5}$ \\ Rose Bosire, ${ }^{5}$ Brandon Guthrie, ${ }^{1,6}$ Romel Mackelprang, ${ }^{6}$ James Kiarie, ${ }^{7,8}$ \\ Grace John-Stewart, ${ }^{1,6,9,10}$ Carey Farquhar ${ }^{1,6,10}$
}

For numbered affiliations see end of article.

\section{Correspondence to Alison C Roxby, Assistant Professor, 325 Ninth Avenue, University of Washington, P.O Box 359909, Seattle, WA 98104-2499 USA; aroxby@u. washington.edu}

LBY and ACR contributed equally.

Received 10 April 2015 Revised 3 November 2015 Accepted 30 December 2015 Published Online First 10 February 2016
To cite: Roxby AC, BenYoussef L, Marx G, et al. J Fam Plann Reprod Health Care 2016;42:264-270.

\begin{abstract}
Background World Health Organization (WHO) guidelines recommend dual contraceptive method use with condoms and another contraceptive to reduce both incidence of HIV/ sexually transmitted infection transmission and unintended pregnancies. This qualitative study assessed the barriers to and motivations for dual contraceptive use in Kenyan HIV-serodiscordant couples.
\end{abstract}

Methods HIV-serodiscordant couples in Nairobi, Kenya, were recruited from two longitudinal cohorts. Qualitative semistructured interviews were conducted using a semistructured questionnaire. Twelve male and 12 female members of serodiscordant couples and 10 women with incident pregnancies during the cohort studies were included.

Results Few couples reported using dual contraceptive methods, with men reporting more condom use than women. No HIV-seropositive men or HIV-seronegative women reported using non-condom contraception. Men and women agreed that men play a dominant role in decisions to use both condoms and contraception in HIVserodiscordant couples. Participants reported that perceptions of side effects, male partner preference, and reproductive desire were critical factors in contraceptive decisions. Both men and women saw dual contraceptive method use as redundant and a sign of possible unfaithfulness. Many participants actively desired pregnancy, but few were able to accurately define monthly fertility windows.

Conclusions Dual contraceptive method use was low in these HIV-serodiscordant couples, with some couples finding it unnecessary while using condoms, and others being more focused on conceiving a child. Biomedical HIV prevention, including male circumcision, pre-exposure prophylaxis or antiretroviral therapy to reduce HIV transmission, may be more acceptable strategies to promote safer sexual relations among HIVserodiscordant couples and safer conception when desired.

\section{Key message points}

Despite counselling about dual contraceptive use, most HIV-serodiscordant Kenyan couples did not use both condoms and another modern contraceptive.

- Pregnancy desire reduces both contraceptive and condom use among HIV-serodiscordant Kenyan couples and leads to unprotected high-risk intercourse.

- Biomedical HIV prevention (circumcision, treatment as prevention or preexposure prophylaxis) could be key strategies for safer conception and reducing HIV transmission in HIV-serodiscordant couples.

\section{INTRODUCTION}

Globally, it is estimated that nearly half of all new HIV infections occur in longterm, stable heterosexual couples. ${ }^{1}$ In Kenya, 260000 married or cohabiting couples are in an HIV-serodiscordant relationship, and it is estimated that $44.1 \%$ of new HIV infections in Kenya occur between stable couples. ${ }^{2}{ }^{3}$ The World Health Organization (WHO) recommends use of dual contraceptive methods to protect against HIV/sexually transmitted infections and unintended pregnancies among HIV-serodiscordant couples. ${ }^{4}$

Dual contraceptive method use is defined as use of both a condom and an additional highly effective contraceptive method. While 'perfect' condom use alone can provide contraception, typical condom use results in a 1-year cumulative incidence of unintended pregnancy of nearly $15 \% .^{5}$ Other contraceptive 
methods (e.g. intrauterine devices, hormonal contraceptives) are more effective at preventing unintended pregnancies, ${ }^{6}$ but do not protect against transmission of HIV or other sexually transmitted infections; therefore use of dual contraceptive methods is recommended for preventing both HIV/STI transmission and unintended pregnancies within HIV-serodiscordant couples. ${ }^{5}{ }^{6}$ Although serodiscordant couples who participate in voluntary counselling and testing have shown sustained, but imperfect, condom use, ${ }^{7}$ dual contraceptive method use in Kenya remains low. 89

Within two large HIV-serodiscordant couple cohorts in Kenya, ${ }^{10}{ }^{11}$ where individuals had disclosed their HIV status within the couple, a high proportion of women $\left(9.7 \% 0^{10}\right.$ and $15.3 \%$ over 2 years ${ }^{12}$ ) became pregnant despite regular counselling on both condom and non-condom contraceptive method use, provision of free condoms, and access to family planning services. Previous studies have investigated determinants of condom use alone, ${ }^{13}$ but little is understood about determinants of dual contraceptive method use, and there are few published qualitative studies from serodiscordant couples. Among HIV-serodiscordant couples, desire for children, perceptions of hormonal contraception, and the HIV-seropositive partner's gender are possible influences on dual contraceptive method use. ${ }^{13}$ Studying dual contraceptive method use is also important since pregnancies in serodiscordant couples, especially those not using antiretroviral therapy (ART), are evidence of risk behaviour that might result in HIV transmission. We designed a qualitative study using semistructured interviews to assess the barriers and motivations for dual contraceptive method use among Kenyan HIV-serodiscordant couples recruited in the Nairobi area.

\section{METHODS}

\section{Study recruitment}

Study participants were consenting HIV-serodiscordant couples already enrolled in one of two separate cohorts: $\mathrm{CAT}^{10}$ and pre-exposure prophylaxis. ${ }^{14}$ Serodiscordant couples were eligible for these studies if the HIV-seropositive partner had not started ART and did not have advanced HIV (WHO stage IV). Exclusion criteria included pregnancy at enrolment. Couples had to report having intercourse at least once per month and be planning to continue the relationship for 2 years. In Kenya at the time this study was conducted, only HIV-seropositive persons with advanced HIV (CD4 <250) were started on ART; therefore the HIV-seropositive participants in this research were not taking ART. In May 2009, a random list of all eligible couples enrolled in the parent trials was generated, and couples were invited in sequence to participate. Both partners in the couples were interviewed separately until 24 interviews were completed. A random list of women who became pregnant while enrolled in the parent studies was generated, and 10 pregnant women were invited to participate. Interviewers determined that saturation was achieved with these 34 interviews.

The Institutional Review Board of the University of Washington and the Ethics and Scientific Research Committee of the University of Nairobi and Kenyatta National Hospital approved the study procedures and semistructured questionnaires. Participants provided written, informed consent before interviews and were reimbursed 600 Kenyan shillings (US\$7) for their involvement.

\section{Study procedures}

Trained research assistants used semistructured questionnaires to conduct interviews in Kiswahili or English, depending on participant preference. Male and female members of couples were interviewed separately. Pregnant women were interviewed without their partners. Interview questions focused on: partner relationship dynamics; reproductive desire, including the influence of HIV status and knowledge of safer conception strategies; contraceptive knowledge and practices; condom use; and sexual partners. The interviews were digitally audiorecorded, translated into English if in Kiswahili, and transcribed with independent quality checks.

\section{Qualitative data analysis}

After interviews were transcribed, a common inductive approach to coding based on a grounded theory approach was used to analyse the qualitative data. After each interview had been read through several times, analysis proceeded with development of codes to describe the data, focusing on themes of fertility, pregnancy, contraception, and condom use for both contraception and HIV protection. The codes were developed using a constant comparison of data through open coding, axial coding and selective coding to identify themes and experiences revealed during the semistructured interviews. ${ }^{15}$ Two investigators (LB-Y and GM) worked to create the original codes and conduct the initial coding. A third investigator (FK) then used this codebook to code the data. The investigators compared codes for consistency, addressed any discrepancies, and mapped larger themes.

\section{RESULTS}

\section{Study participants}

In total, 34 individuals participated; this included 12 couples, in which there were 5 male and 7 female HIV-seropositive index partners (Table 1). In addition, 4 HIV-seronegative and 6 HIV-seropositive women who became pregnant during the parent studies were interviewed. No HIV-seropositive men or HIV-seronegative women reported using non-condom contraception (Table 2). All HIV-seropositive men 
Table 1 Description of 34 in-depth interview participants

\begin{tabular}{lcc}
\hline Participant description & Number (\%) & HIV serostatus \\
\hline Women, not pregnant & $7(20.5)$ & Seropositive \\
Women, not pregnant & $5(14.7)$ & Seronegative \\
Men & $5(14.7)$ & Seropositive \\
Men & $7(20.5)$ & Seronegative \\
Pregnant women & $6(17.6)$ & Seropositive \\
Pregnant women & $4(11.7)$ & Seronegative \\
\hline
\end{tabular}

reported $100 \%$ condom use; no seropositive or seronegative women reported 100\% condom use. Only three out of seven HIV-seropositive women reported any contraception use. Condom use was reported more than non-condom contraceptive use among all participants.

\section{Barriers to and motivations for dual contraceptive method use}

Four key influencing themes regarding barriers to and motivations for dual contraceptive method use emerged in this study: condom use, non-condom contraceptive method use, dual contraceptive method use, and pregnancy desire.

Theme 1: condom use

Condom use among HIV-serodiscordant couples varied between the individually interviewed cohorts, with HIV-seronegative women reporting the least condom use and HIV-seropositive men (partners of those same women) reporting the most. The majority of pregnant participants reported that their partner had refused any condom use despite knowing the risk of HIV transmission: "It's only me who forced him which results in my disagreement with him and because he is a man, at times he defeats me in my struggles and has sex with me without a condom" (HIV-seropositive pregnant female). A minority of non-pregnant women noted inconsistent condom use due to pregnancy desire. HIV acquisition was overtly mentioned as a risk many were willing to take to conceive a child: "But sometimes if we want a child, we say let's not use condom, let us just do without" (HIV-seronegative non-pregnant woman).
Both men and women expressed concern that condom use interfered with the emotional aspects of sexual encounters and decreased sexual pleasure: "If you do it with a condom, it's not good. (laughs).... Because you are used to flesh to flesh but rubber to flesh is not good" (HIV-seronegative non-pregnant female). "Using the condom due to the disease, I know she is not happy the way we are doing it. It's like giving kale and ugali[Kenyan starch dish] to someone who is used to meat and ugali" (HIV-seronegative male).

Some couples were motivated to use condoms for HIV protection, which resulted in perceived less need for another contraceptive method (see below).

Theme 2: non-condom contraceptive method use

Regardless of participant gender, perceived side effects of hormonal contraception were listed as significant barriers to utilisation. In interviews, 22 out of 24 men and non-pregnant women specifically stated that these side effects influenced the use and/or choice of contraceptive method. Among the pregnant women interviewed, all mentioned these adverse effects as deterrents to their use. Perceived side effects included decreased libido, malignancy, birth defects, weight gain or loss, malaise, hypertension, skin manifestations, and infertility following hormonal contraception use: "The fears are... if one was using [contraceptives], one may give birth to a baby who might have deformities" (HIV-seropositive female). "Some women develop abnormal growth in the uterus which discourages many women ... and the fact that you may not get a child when you want, even after you quit using them" (HIV-seronegative pregnant female). Participants cited their personal experiences and those of friends, relatives and counsellors.

Among the pregnant women interviewed, male partner disapproval was reported as a barrier to noncondom contraceptive use for six of nine of them: "My husband doesn't trust them, because he says they are not reliable" (HIV-seropositive pregnant female). "It's because my partner convinced me that since he was using the condoms-thus there was no need of

Table 2 Reported use of condoms, contraception and the dual contraceptive method among male and non-pregnant female participants

\begin{tabular}{|c|c|c|c|c|}
\hline \multirow[b]{2}{*}{ Reported use } & \multicolumn{2}{|c|}{ Couple: man HIV-seropositive } & \multicolumn{2}{|c|}{ Couple: woman HIV-seropositive } \\
\hline & Men $(n=5)$ & Women $(n=5)$ & Men $(n=7)$ & Women $(n=7)$ \\
\hline \multicolumn{5}{|l|}{ Condoms } \\
\hline Consistent & $5(100 \%)$ & $3(60 \%)$ & $6(86 \%)$ & $6(86 \%)$ \\
\hline Inconsistent & 0 & $2(40 \%)$ & $1(14 \%)$ & $1(14 \%)$ \\
\hline Contraceptives & 0 & 0 & $3(43 \%)$ & $3(43 \%)$ \\
\hline Dual contraceptive (condom and contraception) & 0 & 0 & $3(43 \%)$ & $3(43 \%)$ \\
\hline
\end{tabular}

Consistent condom use was defined as 'with every act of intercourse'. Inconsistent condom use was defined as 'sometimes using a condom, but not $100 \%$ '. Contraceptives were defined as 'any highly effective method'; those available in Kenya at this time include combined oral contraceptives, depot medroxyprogesterone acetate (DMPA) injections, progestin-only pills, copper intrauterine devices, implants, and tubal ligation. 
me using injection and contraceptive pills" (HIV-seronegative pregnant female).

Current or potential pregnancy desire strongly influenced non-condom contraception use. Two-thirds of pregnant women interviewed reported not using contraception because they wanted a child. Among non-pregnant women, only 4 of 16 women cited a desire for children as the reason for not using hormonal contraception. Other reasons included community opposition: "He[her husband] talked with other people about contraception... those old men told him if your wife uses contraception, let her go...sometimes women in our neighbourhood, they tell him maybe God placed one child only in the womb so if you family plan, how will you get that baby" (HIV-seronegative pregnant female).

Men also described strong fertility desires, and these were an important barrier to family planning use, especially in partnerships with no children or few children: "To me a person who family plan has a family already...This is a childless marriage, so we can't family plan. I only use the condom because of our status, otherwise I can't talk of contraception with this particular" (HIV-seronegative male).

\section{Theme 3: dual contraceptive method use}

The majority of participants noted that condom use alone negated the need of any other contraception. Participants felt that condoms provided adequate protection against both HIV and pregnancy: "she was using a contraceptive pill before we realized ...our status, but now it is irrelevant ... because now condom does everything. She is not using anything" (HIV-seronegative male).

Furthermore, many men and even some women interpreted the concept of dual contraceptive methods as evidence of unfaithfulness "I decided to abandon it [hormonal contraception] because we are now using the condom and I don't have any other relationship outside my marriage" (HIV-seronegative female). "My opinion is to continue using condom. There is no need of using any other method. If I do that, it's like being ready to have extra-marital affair, which is not good" (HIV-seronegative female).

Dual contraceptive use was only reported among six individual participants, including three HIV-seronegative men and their three HIV-seropositive, non-pregnant partners. These individuals reasoned that, while condom use strongly protected against HIV transmission, it was less likely to protect against an unintended pregnancy. Two of the HIV-seropositive women reported concern that their partners would intentionally compromise the condoms: "Men are crooks, he can even prick it. I carefully monitor him and keep the torch nearby so that he does not prick it.... I feel I would rather use the injection together with the condom."

HIV-seronegative men also justified the use of dual contraception because of concern that the condom would unintentionally break. One stated, "You can use this condom and find that it's broken and maybe the lady was fertile, then she becomes pregnant. Then it becomes a problem twice, the condom is broken and she becomes pregnant."

\section{Theme 4: desire for pregnancy}

Desire for pregnancy strongly influenced the consistent use of condoms, and those participants wanting children would often abstain from condom use in order to conceive, even with knowledge of HIV transmission risk. As one HIV-seronegative participant explained her planned pregnancy, "Yes, because immediately after my periods we met, and that's when I conceived. We purposely decided against the use of condom during that time... I was extremely happy though concerned-I was scared I may have contracted HIV when we failed to use a condom."

Participants recognised and understood that there were options available to minimise periconception HIV transmission risk (at the time of this study, proven techniques were limited to sperm washing and assisted reproductive technology), but these were viewed as too costly to be relevant to their own situation: "The only one I can remember is the one involving extracting sperms, washing them, then testing it and put it inside a woman to fertile, though we ... understand it is very expensive" (HIV-seronegative pregnant woman). Home insemination to protect male HIV-seronegative partners was not mentioned by any participants as an option.

Participants reported being counselled to try for pregnancy during fertile windows. Assuming a 28-day menstruation cycle with peak fertility days occurring between days 9 and $14,{ }^{16}$ only $15.2 \%(5 / 33)$ of the interviewed participants had an understanding of their optimal fertility window (Figure 1), although some reported that they practised timed unprotected sexual intercourse within their perceived 'fertile' time frame (Figure 1): "I was told that if you understand the women's cycle well, once in a while you can have sex without condom for the sake of looking for a child... I don't think the two days I haven't used will affect in any way" (HIV-seronegative male). Many participants described wrongly that their peak conception time occurred in the days leading up to menstruation: "Ok I know it's maybe when I'm almost getting my periods, like 4-5 days before" (HIV-seropositive pregnant female). "It is before her period starts. Around 5 days before..... We try to conceive one day in those 5 days without using the condom. According to the advice given by the doctor I see like those 5 days are so many I might infect instead of impregnating her. So we just try our luck" (HIV-seropositive male).

\section{DISCUSSION}

This qualitative study interviewed men, women and pregnant women enrolled in cohorts of 


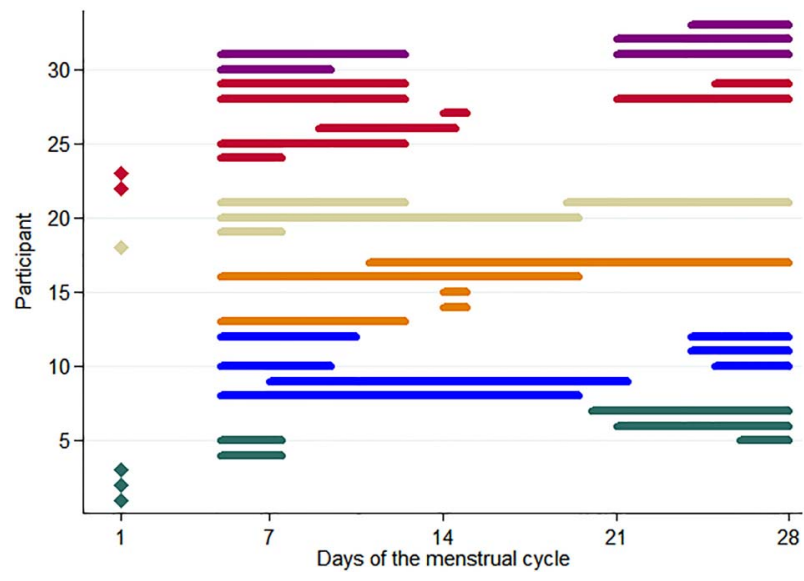

Figure 1 Participant description of the optimal fertile window during the menstrual cycle. Menstruation is defined as days $1-5$; the peak or optimal fertile window is defined as days 9-14 within a 28-day cycle. Of the 34 participants interviewed, 33 responded to the question and are shown in the figure; one was not queried. Diamonds represent the six participants who were unable to define a fertility window. Purple bars represent HIV-seropositive pregnant women; red bars represent HIV-seropositive non-pregnant women; tan bars represent HIV-seronegative pregnant women, orange bars represent HIV-seronegative non-pregnant women; blue bars represent HIV-seropositive men; green bars represent HIV-seronegative men.

HIV-serodiscordant couples in Nairobi, Kenya. Our interviews found that few participants were adhering to current guidelines to consistently use condoms, despite receiving extensive couple-based HIV-prevention counselling as part of research participation, and even fewer appeared to endorse dual contraception practices for family planning. Within couples where the man was HIV-seropositive, men appeared to over-report their condom use at $100 \%$, while their seronegative female partners reported much lower condom use. Men were cited as the dominant decision-maker regarding both condoms and non-condom contraception use in the couple, and perceptions of adverse effects, male partner preferences, and pregnancy desire were critical factors in the decision to use non-condom contraception. Dual contraceptive method use, despite being a specific recommendation to cohort study participants, was seen by many as unnecessary and a potential indication of unfaithfulness. Many couples intentionally did not use condoms or any other contraceptive method out of a desire for conception, and knowledge of fertile windows to optimally time intercourse was poor.

Our research suggests that commonly implemented prevention messages, such as consistent condom and dual contraceptive method use, are not accepted by many Kenyan HIV-serodiscordant couples. Despite specific awareness of their own risk of HIV transmission and acquisition, some couples chose not to use condoms because they desired children. Even among those wanting contraception, myths about highly effective family planning methods were common, especially regarding poor effectiveness and dangerous side effects. Condoms were often relied upon for contraception despite their lower effectiveness.

These data were collected in 2009 , when condoms were the sole recommended HIV prevention message for serodiscordant couples, and when ART use was restricted to the sickest patients. Recommendations for couples to always use condoms are evolving in the face of new prevention modalities, and the realisation that, for many in sub-Saharan Africa, desire for pregnancy may outweigh fear of HIV transmission. ${ }^{13}{ }^{17}$ Given the barriers to regular condom use in HIV-serodiscordant couples that our study and others ${ }^{18}$ have observed, biomedical prevention including use of PrEP and ART may be more acceptable strategies for reducing HIV transmission in HIV-serodiscordant couples, especially since condom use by couples over long periods of time may be unrealistic. Expanding the use of ART to HIV-seropositive partners in heterosexual HIV-serodiscordant couples could result in approximately $80-92 \%$ reduction in HIV transmission in the partnership in real world settings. ${ }^{19}$ Male circumcision $^{20}$ and PrEP in HIV-seronegative individuals ${ }^{11}$ significantly reduces HIV transmission within HIV-serodiscordant couples, and WHO recommends considering daily oral PrEP for uninfected partners in serodiscordant couples. ${ }^{21}$ Further, PrEP and ART could result in safer conception with less chance of perinatal HIV transmission, and adherence to both interventions may be enhanced in the peripregnancy period. Recent research supports the use of PrEP as it could be a promising option for serodiscordant couples desiring children. ${ }^{17}{ }^{22-24}$ While timed vaginal intercourse could be feasible in developing country settings, our data show that knowledge of fertility timing is low; a recent study in Kenya concluded that reliance on fertility windows was unlikely to be effective without extra support such as home visits and condom provision. ${ }^{25}$ 'Preconception clinics' where comprehensive counselling and services are offered to help serodiscordant couples review their options may be an appropriate way for couples to learn about the many options available to them for safer conception.

All participants in this study received extensive, sensitive counselling appropriate for serodiscordant couples as well as access to support groups. This study highlights that, for some couples, counselling alone is probably insufficient to change behaviours and attitudes not supported by the larger cultural environment. Many participants reported that condom use and dual contraceptive method use were not accepted in their families or communities. In addition, both women and men in this study agreed that men are the primary decision-maker for both contraception and condom use in the couple; therefore even among mutually disclosed HIV-serodiscordant couples, 
women's subordinate role in society and in intimate relationships represents an ongoing challenge for condom and contraceptive use. A conceptual framework developed by Crankshaw et $a l^{26}$ to understand HIV risk behaviour and fertility goals in HIV-serodiscordant couples outlined that reproductive health-decision-making is challenging, since each member of the couple may have different fertility and reproductive health goals, different risks, and different power in the relationship. Using this conceptual framework, the authors argue that the periconception period can serve as an 'entry point' to engage both members of a couple in reproductive health-decisionmaking. A recent systematic review of behavioural interventions to promote dual contraceptive method use did not identify rigorously proven behavioural methods to promote dual contraceptive method use, indicating a need for research to identify effective ways of promoting dual contraception. ${ }^{27}$

Our study was limited by our volunteer participants, who may have been more comfortable discussing this topic than others. Study generalisability may be limited, since participants were enrolled in longitudinal studies that provided regular HIV risk reduction education, and required that partners mutually disclose their HIV status. Partners in relationships who have not mutually disclosed their HIV status probably face different barriers to dual contraceptive method use. Participants had been enrolled in parent studies for different lengths of time and may have had different exposure to counselling messages.

In conclusion, despite receiving intensive, couplebased HIV-prevention education, most men and women in HIV-1-serodiscordant couple cohorts in Kenya did not adopt recommendations to use dual contraceptive methods. Participants identified numerous barriers to adopting these practices in their daily lives, and voiced desires for pregnancy which were not addressed by the counselling and services provided in the discordant couple clinic. This study highlights the need for further action to identify strategies to reduce unprotected sex in HIV-serodiscordant couples and to promote safer conception when pregnancy is desired.

\footnotetext{
Author affiliations

${ }^{1}$ Assistant Professor, Departments of Medicine and Global Health, University of Washington, WA, USA

${ }^{2}$ Resident Physician, Department of Emergency Medicine, Alameda County Medical Center, Highland Hospital, Oakland, CA, USA, and University of Washington School of Medicine, WA, USA

${ }^{3}$ Infectious Disease Fellow, Department of Medicine, University of Colorado, Aurora, CO, USA

${ }^{4}$ Physician, Kenya Medical Research Institute, Nairobi, Kenya

${ }^{5}$ Senior Clinical Research Scientist, Kenya Medical Research Institute, Nairobi, Kenya
}

${ }^{6}$ Assistant Professor, Departments of Epidemiology and Global Health, University of Washington, Seattle WA, USA

${ }^{7}$ Acting Instructor, Department of Global Health, University of Washington, Seattle, WA, USA

${ }^{8}$ Professor, Department of Obstetrics and Gynaecology, University of Nairobi, Nairobi, Kenya and Department of Obstetrics and Gynaecology, Kenyatta National Hospital, Nairobi, Kenya ${ }^{9}$ Professor, Departments of Medicine, Global Health, Pediatrics, and Epidemiology, University of Washington, Seattle, WA, USA

${ }^{10}$ Professor, Departments of Medicine, Epidemiology, Global Health, University of Washington, Seattle, WA, USA

Acknowledgements We thank the following: the clinic and data management teams in Nairobi, Kenya; Dr Emily Vogtmann; and Ken Tapia of the University of Washington CFAR

Biostatistics Core for assistance with the figure. We gratefully acknowledge our study participants.

Funding This research was supported by the UW Center for AIDS Research (CFAR), funded by the National Institutes of Health (NIH) (P30 AI027757), K24 AI087339, K23HD071788, K01AI098527, R01HD23412, and the American Relief and Recovery Act. LBY, ACR, RB were supported by the Fogarty International Clinical Research Scholars and Fellows Program funded under NIH Fogarty International Center grant R24 TW000007; LBY, GM and RB were also supported by the International Clinical Research Fellows Program at Vanderbilt University, R24 TW007988; and LBY received support from the University of Washington Global Health Opportunities Fellowship. GM was supported by a PReP Junior Investigator award. The parent cohort studies were funded by US National Institutes of Health (NIH) R01 AI068431 and by the Bill and Melinda Gates Foundation (\#47674).

Competing interests None declared.

Ethics approval Institutional Review Board at University of Washington and Ethical Review Committee at the University of Nairobi, Nairobi, Kenya.

Provenance and peer review Not commissioned; externally peer reviewed.

\section{REFERENCES}

1 National AIDS Control Council of Kenya. United Nations General Assembly Special Session on HIV and AIDS: Country Report, Kenya, 2010.

2 Kenya AIDS Strategic Framework 2014/2015-2018/2019. Nairobi, Kenya, 2014.

3 National AIDS and STI Control Programme, Ministry of Health, Kenya. July 2008. Kenya AIDS Indicator Survey 2007. Nairobi, Kenya.

4 WHO. Hormonal Contraception and HIV: Technical Statement. Geneva: World Health Organization, 2012.

5 Hatcher RA. Contraceptive Technology. 19th rev. edn. New York, NY: Ardent Media, 2007:xxx, 874 p.

6 Cates W Jr, Steiner MJ. Dual protection against unintended pregnancy and sexually transmitted infections: what is the best contraceptive approach? Sex Transm Dis 2002;29:168-174.

7 Allen S, Meinzen-Derr J, Kautzman M, et al. Sexual behavior of HIV discordant couples after HIV counseling and testing. AIDS 2003;17:733-740.

8 Imbuki K, Todd CS, Stibich MA, et al. Factors influencing contraceptive choice and discontinuation among HIV-positive 
women in Kericho, Kenya. Afr J Reprod Health 2010;14(4 Spec no.):98-109.

9 Mutiso SM, Kinuthia J, Qureshi Z. Contraceptive use among HIV infected women attending Comprehensive Care Centre. East Afr Med J 2008;85:171-177.

10 Guthrie BL, Choi RY, Bosire R, et al. Predicting pregnancy in HIV-1-discordant couples. AIDS Behav 2010;14:1066-1071.

11 Baeten JM, Donnell D, Ndase P, et al. Antiretroviral prophylaxis for HIV prevention in heterosexual men and women. N Engl J Med 2012;367:399-410.

12 Ngure K, Heffron R, Mugo NR, et al. Contraceptive method and pregnancy incidence among women in HIV-1serodiscordant partnerships. AIDS 2012;26:513-518.

13 Ngure K, Mugo N, Celum C, et al. A qualitative study of barriers to consistent condom use among HIV-1 serodiscordant couples in Kenya. AIDS Care 2012;24:509-516.

14 Mujugira A, Baeten JM, Donnell D, et al. Characteristics of HIV-1 serodiscordant couples enrolled in a clinical trial of antiretroviral pre-exposure prophylaxis for HIV-1 prevention. PLOS ONE 2011;6:e25828.

15 Corbin JM, Strauss AL, Strauss AL. Basics of Qualitative Research: Techniques and Procedures for Developing Grounded Theory. 3rd edn. Los Angeles, CA: Sage Publications, Inc., 2008.

16 Wilcox AJ, Dunson D, Baird DD. The timing of the "fertile window" in the menstrual cycle: day specific estimates from a prospective study. BMJ 2000;321:1259-1262.

17 Matthews LT, Smit JA, Cu-Uvin S, et al. Antiretrovirals and safer conception for HIV-serodiscordant couples. Curr Opin HIV AIDS 2012;7:569-578.

18 Church K, Wringe A, Fakudze P, et al. Reliance on condoms for contraceptive protection among HIV care and treatment clients: a mixed methods study on contraceptive choice and motivation within a generalised epidemic. Sex Transm Infect 2014;90:394-400.
19 Curran K, Baeten JM, Coates TJ, et al. HIV-1 prevention for HIV-1 serodiscordant couples. Curr HIV/AIDS Rep 2012;9:160-170.

20 Bailey RC, Moses S, Parker CB, et al. Male circumcision for HIV prevention in young men in Kisumu, Kenya: a randomised controlled trial. Lancet 2007;369:643-656.

21 World Health Organization. Guidance on Ral Pre-Exposure Prophylaxis (PrEP) for Serodiscordant Couples, Men and Transgender Women Who Have Sex with Men at High Risk of HIV: Recommendations for Use in the Context of Demonstration Projects. Geneva, Switzerland, 2012.

22 Whetham J, Taylor S, Charlwood L, et al. Pre-exposure prophylaxis for conception (PrEP-C) as a risk reduction strategy in HIV-positive men and HIV-negative women in the UK. AIDS Care 2014;26:332-336.

23 Mugo NR, Hong T, Celum C, et al. Pregnancy incidence and outcomes among women receiving preexposure prophylaxis for HIV prevention: a randomized clinical trial. JAMA 2014;312:362-371.

24 Matthews LT, Heffron R, Mugo NR, et al. High medication adherence during periconception periods among HIV-1-uninfected women participating in a clinical trial of antiretroviral pre-exposure prophylaxis. J Acquir Immune Defic Syndr 2014;67:91-97.

25 Mmeje O, van der Poel S, Workneh M, et al. Achieving pregnancy safely: perspectives on timed vaginal insemination among HIV-serodiscordant couples and health-care providers in Kisumu, Kenya. AIDS Care 2015;27:10-16.

26 Crankshaw TL, Matthews LT, Giddy J, et al. A conceptual framework for understanding HIV risk behavior in the context of supporting fertility goals among HIV-serodiscordant couples. Reprod Health Matters 2012;20(39 Suppl):50-60.

27 Lopez LM, Stockton LL, Chen M, et al. Behavioral interventions for improving dual-method contraceptive use. Cochrane Database Syst Rev 2014;3:CD010915.

\section{FACULTY OF SEXUAL \& REPRODUCTIVE HEALTHCARE MEMBERSHIP EXAMINATION}

The Membership Examination (MFSRH) consists of:

\section{Part 1 Single Best Answer paper (SBA)}

London-based examination held annually in April and October. Applications for April 2017 examination must be received by January 2017. The syllabus for the Part 1 examination is on the FSRH website.

\section{$\square$ Evidence Based Commentary (EBC)}

Candidates can view the released topic and candidate guidance notes on the FSRH website. (Please note you will now need to register on the new FSRH website before accessing this information.) There is an absolute deadline of $\mathbf{3 1}$ August 2017 to submit the EBC on this topic. You can submit an EBC before applying to enter the Part 1 examination.

\section{- Part 2 Examination (CRQ, EMQ, OSCE)}

Applications for the MFSRH Part 2 to be held June 2017 must be received by January 2017.

Further information and the Part 2 Syllabus are on the FSRH website. The qualification is subject to re-certification every 5 years. For the current MFSRH Examination Regulations, information on all components of the MFSRH examination and application forms, please visit the Faculty of Sexual and Reproductive Healthcare Website: https://www.fsrh.org/careers-andtraining/membership-exams/ or contact examsofficer@fsrh.org 